\title{
Efficacy and safety of World Health Organization group 5 drugs for multidrug-resistant tuberculosis treatment
}

\author{
Nicholas Winters ${ }^{1,2}$, Guillaume Butler-Laporte ${ }^{1}$ and Dick Menzies ${ }^{1,2}$ \\ Affiliations: \\ ${ }^{1}$ Respiratory Epidemiology and Clinical Research Unit, Montreal Chest Institute, McGill University, Montreal, \\ QC, Canada. \\ ${ }^{2}$ Dept of Epidemiology and Biostatistics, McGill University, Montreal, QC, Canada. \\ Correspondence: \\ Dick Menzies, Montreal Chest Institute, Room K1.24, 3650 St Urbain, Montreal, QC, Canada, H2X 2P4. \\ E-mail: Dick.Menziesamcgill.ca
}

ABSTRACT The efficacy and toxicity of several drugs now used to treat multidrug-resistant tuberculosis (MDR-TB) have not been fully evaluated.

We searched three databases for studies assessing efficacy in MDR-TB or safety during prolonged treatment of any mycobacterial infections, of drugs classified by the World Health Organization as having uncertain efficacy for MDR-TB (group 5).

We included 83 out of 4002 studies identified. Evidence was inadequate for meropenem, imipenem and terizidone. For MDR-TB treatment, clarithromycin had no efficacy in two studies (risk difference (RD) $-0.13,95 \%$ CI $-0.40-0.14$ ) and amoxicillin-clavulanate had no efficacy in two other studies (RD 0.07, 95\% CI -0.21-0.35). The largest number of studies described prolonged use for treatment of nontuberculous mycobacteria. Azithromycin was not associated with excess serious adverse events (SAEs). Clarithromycin was not associated with excess SAEs in eight controlled trials in HIV-infected patients (RD 0.00, 95\% CI -0.02-0.02), nor in six uncontrolled studies in HIV-uninfected patients, whereas six uncontrolled studies in HIV-infected patients clarithromycin caused substantial SAEs (proportion 0.20, 95\% CI 0.12-0.27).

For most group 5 drugs we found inadequate evidence of safety for prolonged use or for efficacy for MDR-TB, although macrolides appeared to be safe in prolonged use.

@ERSpublications

Weak evidence for the efficacy and safety of group 5 drugs for MDR-TB suggests they should be evaluated using RCTs http://ow.ly/P8I3t

This article has supplementary material available from erj.ersjournals.com

Received: April 242015 | Accepted after revision: June 22 2015 | First published online: Sept 172015

Support statement: This study was supported by funding provided by the World Health Organization. Funding information for this article has been deposited with FundRef.

Conflict of interest: Disclosures can be found alongside the online version of this article at erj.ersjournals.com

The content of this work is copyright of the authors or their employers. Design and branding are copyright @ERS 2015. 


\section{Introduction}

Tuberculosis (TB) is one of the most deadly communicable diseases in the world, causing an estimated 9 million new cases and 1.9 million deaths in 2013 [1]. Of these, an estimated 480000 patients had multidrug-resistant TB (MDR-TB) [1], defined as resistance to at least rifampicin and isoniazid [2], and $\sim 43000$ patients had extensively drug-resistant TB (XDR-TB) [1], defined as MDR-TB with additional resistance to any fluoroquinolone and any second-line injectable [3]. Treatment of MDR-TB is challenging as it is very expensive (the cost to treat each MDR-TB and XDR-TB patient is approximately US $\$ 134000$ and US $\$ 430000$, respectively [4]), requires lengthy use of more toxic second-line anti-TB drugs [5, 6], yet is associated with higher failure and death rates than drug-susceptible TB.

Drugs used in the treatment of MDR- and XDR-TB include amoxicillin-clavulanate (AMX-CLV), meropenem, imipenem-cilastatin, thioridazine, terizidone and the macrolides: clarithromycin and azithromycin [7]. The efficacy and toxicity of these agents are unclear, as there are few published data regarding their use in TB treatment; hence these are considered group 5 drugs by the World Health Organization. Systematic reviews have been published assessing the efficacy and toxicity of linezolid [8-10] and clofazimine $[9,11]$, as well of the safety (only) of terizidone [12] and the efficacy (only) of macrolides [10], but there have been no reviews of the other second-line drugs used for the treatment of MDR-TB.

We have conducted a systematic review and meta-analysis to assess the efficacy and toxicity of AMX-CLV, meropenem, imipenem-cilastatin, thioridazine, terizidone and the macrolides when used for the treatment of MDR- and XDR-TB. Because we were unsure of the number of studies assessing these group 5 drugs when used for MDR- or XDR-TB, we also included studies that assessed the toxicity of these same drugs during prolonged use for prevention or treatment of other mycobacterial infections: the non-tuberculous mycobacteria (NTM).

\section{Methods}

Search strategy

We performed two separate searches, using the MEDLINE, Embase and Cochrane library databases, for studies reporting efficacy or toxicity of AMX-CLV, meropenem, imipenem/cilastatin, thioridazine, terizidone and the macrolides clarithromycin and azithromycin when used for the treatment of microbiologically confirmed Mycobacterium tuberculosis. We also included reports describing toxicity of the same drugs used to treat any mycobacterial disease for $\geqslant 6$ months. We included original research articles describing results of randomised controlled trials (RCTs), cohort or case-control studies published in the peer-reviewed literature between 1946 and October 20, 2014. We also identified relevant articles from the references cited in the retrieved articles and previously published reviews.

We developed comprehensive search strategies separately for efficacy and toxicity using a combination of keywords and search terms. We included all search terms related to tuberculosis and our drugs of interest as exploded medical subject headings (MeSH) and free-text keywords in titles or abstracts. The full search terms for each database and search can be found in the online supplementary material.

\section{Study selection}

We included original articles published in the peer-reviewed literature, written in any language, that described efficacy and toxicity of at least one of the drugs of interest used for the treatment of $\geqslant 20$ human subjects with MDR-TB, or that described toxicity of these drugs during use for $\geqslant 6$ months to prevent or treat NTM infections. The studies of NTM were included as these are mycobacterial infections that require prolonged treatment in both HIV-infected and -uninfected patients. Hence we believed the information regarding toxicity would be potentially applicable to patients treated for MDR- or XDR-TB. Studies of patients who had strictly extrapulmonary MDR-TB were excluded from the analysis of efficacy as treatment outcomes are rarely confirmed bacteriologically, introducing greater uncertainty in estimates of efficacy. Articles were excluded if they were reviews, in vitro, pharmacokinetic or laboratory studies, studies of animals or reported results that overlapped with a previous report. The search results (titles and abstracts, and then full text) were screened for inclusion independently by two authors (N. Winters and G. Butler-Laporte). Differences at each stage were resolved by consensus.

\section{Data abstraction}

Data abstraction was performed by two authors for included studies, using a standardised data abstraction form. We judged quality on two criteria: 1) if treatment outcomes were defined according to Laserson criteria (cured: MDR-TB patients completed treatment as planned and had at least five consecutive negative sputum cultures during the final 12 months of treatment; treatment completed: completed treatment as planned but did not meet the definition for cure due to lack of bacteriological results; failed: if two or more cultures out of the five in the final 12 months were positive, or if one out of the final three 
cultures were positive) [2]; and 2) whether adverse events were classified based on a priori published definitions such as the Common Terminology Criteria for Adverse Events guidelines [13]. All studies were included regardless of whether they met these quality criteria. For each study we recorded data on the average age of the patients, HIV prevalence, the companion drugs and mean number of drugs in the regimen, duration of drug use, dosage, whether the drug treatment was standardised or individualised, duration of follow-up and description of the study design.

\section{Data synthesis}

We estimated efficacy by comparing the proportion with treatment success (cured or completed) among patients with MDR-TB who received one of the drugs of interest with those who did not receive that drug. For toxicity in both MDR-TB and NTM, we abstracted the occurrence of serious adverse events (SAEs), defined as grade 3 or 4 adverse events if reported, or whether the drug of interest was stopped due to adverse events. Risk differences $(\mathrm{RD})$, i.e. the absolute difference in observed risk of SAE between those receiving a treatment and those not receiving it were estimated in controlled studies, meaning data studies reporting the occurrence of SAEs in patients who received and did not receive the drug of interest. In uncontrolled studies we calculated the proportion of patients in whom SAEs occurred that were attributed to the drug of interest.

Random effects models were used to generate pooled effect estimates (RD or proportions) and their 95\% confidence intervals for treatment success and toxicity using $\mathrm{R}$ (version 3.1.0; http://cran.r-project.org/)

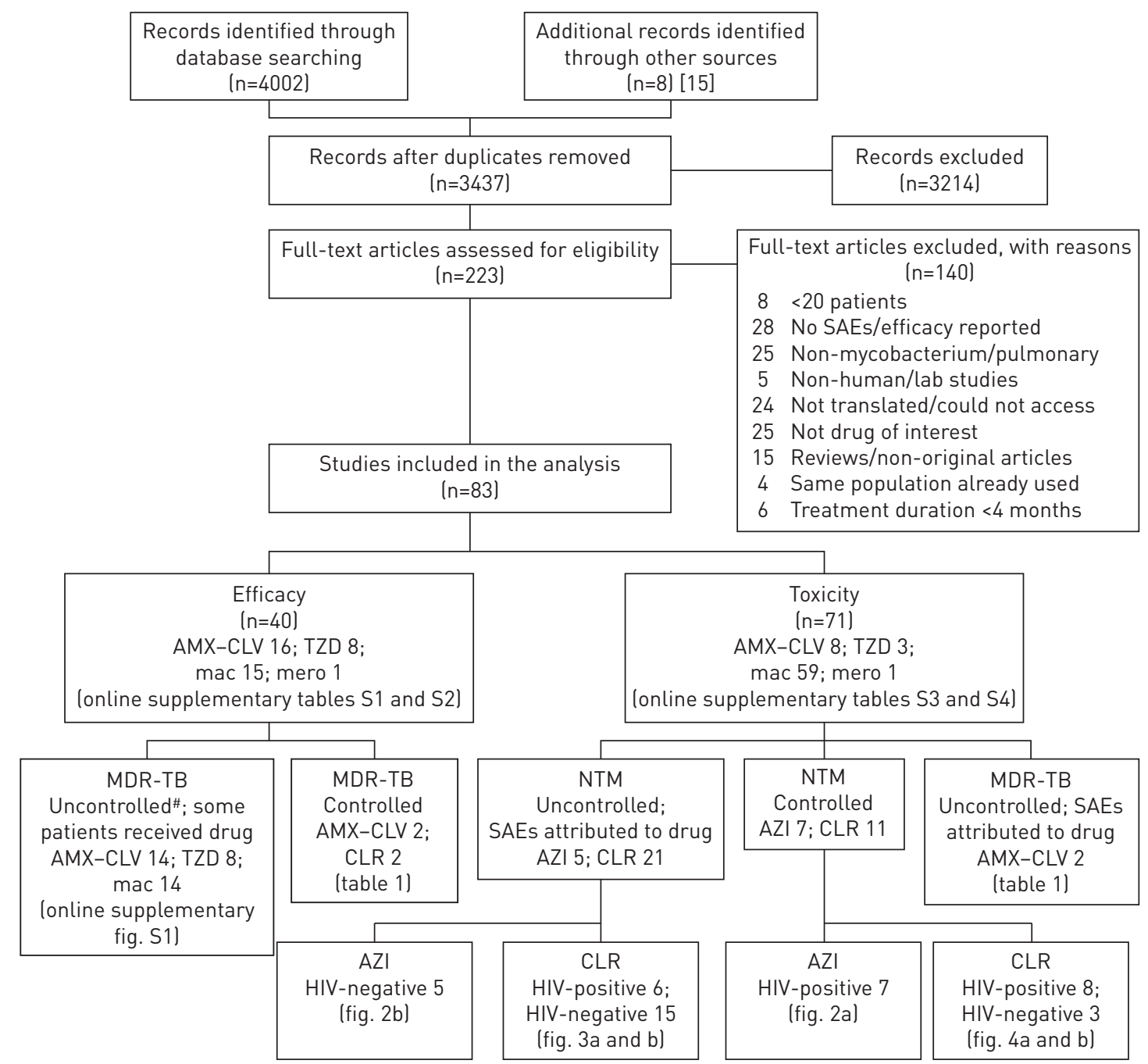

FIGURE 1 Flow diagram of the search strategy and selection of studies, as well as a guide as to in which figure and table the information in each box can be found (meta-analyses were performed on all studies marked controlled or uncontrolled). SAEs: serious adverse events; AMX-CLV: amoxicillin-clavulanate; TZD: terizidone; mac: macrolide; mero: meropenem; MDR-TB: multidrug-resistant tuberculosis; CLR: clarithromycin;

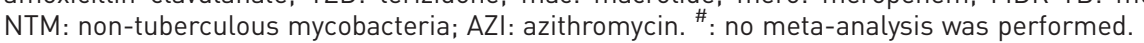


TABLE 1 Summary of results of studies of the efficacy and toxicity of amoxicillin-clavulanate and macrolides used for treatment of non-tuberculous mycobacteria (NTM) and multidrug-resistant tuberculosis (MDR-TB) in HIV-infected and uninfected patients

\begin{tabular}{|c|c|c|c|c|c|c|}
\hline $\begin{array}{l}\text { Type of } \\
\text { design }\end{array}$ & Effect measure & Studies & Patients" & $\begin{array}{c}\text { Range of } \\
\text { outcomes }\end{array}$ & $\begin{array}{l}\text { Heterogeneity } \\
\qquad\left(I^{2}\right) \%\end{array}$ & $\begin{array}{l}\text { Pooled estimate } \\
\qquad(95 \% \mathrm{CI})\end{array}$ \\
\hline
\end{tabular}

\begin{tabular}{|c|c|c|c|c|c|c|c|}
\hline \multicolumn{8}{|l|}{ NTM SAEs } \\
\hline \multicolumn{8}{|l|}{ HIV-infected } \\
\hline Clarithromycin & Controlled & Risk difference & 8 & 1088/1111 & $0.00-0.02$ & 0 & $0.00(-0.02-0.02)$ \\
\hline Clarithromycin & Uncontrolled & Proportion & 6 & 584 & $0.06-0.27$ & 82.1 & $0.20(0.12-0.27)$ \\
\hline \multicolumn{8}{|l|}{ HIV-uninfected } \\
\hline Clarithromycin & Controlled & Risk difference & 3 & $174 / 175$ & $-0.01-0.11$ & 0 & $0.01(-0.06-0.07)$ \\
\hline Clarithromycin & Uncontrolled & Proportion & 15 & 615 & $0.00-0.22$ & 64.5 & 0.04 (0.02-0.07) \\
\hline \multicolumn{8}{|l|}{$\begin{array}{l}\text { MDR-TB } \\
\text { HIV-uninfected }\end{array}$} \\
\hline \multicolumn{8}{|l|}{ Efficacy } \\
\hline AMX-CLV & Controlled & Risk difference & 2 & $73 / 272$ & $-0.02-0.31$ & 43.6 & $0.07(-0.21-0.35)$ \\
\hline Clarithromycin & Controlled & Risk difference & 2 & $86 / 257$ & -0.29 to -0.02 & 65.9 & $-0.13(-0.40-0.14)$ \\
\hline \multicolumn{8}{|l|}{ SAEs } \\
\hline AMX-CLV & Uncontrolled & Proportion & 3 & 77 & $0.04-0.60$ & 71.5 & $0.12(0.00-0.28)$ \\
\hline
\end{tabular}

with the package meta version 4.0-2 (http://CRAN.R-project.org/package=meta). We stratified analyses based on whether outcomes were reported in patients who received or did not receive the drug of interest (RCTs or controlled cohorts) or were reported without comparison groups (case series). Additionally, we stratified analyses by the HIV status of the subjects and by MDR-TB versus NTM for toxicity. We assessed heterogeneity of studies by using the coefficient of inconsistency $\left(I^{2}\right)$ and its $95 \%$ confidence interval [14].

\section{Results}

As seen in figure 1, 4002 potentially relevant titles were identified: 657 from MEDLINE, 3613 from Embase and 17 from the Cochrane Library. Eight citations were included from the references of a previously published systematic review by Hwang et al. [15]. After removing duplicates we screened 3437 studies, 3214 of which were excluded during title and abstract review. A further 140 of the 223 full texts reviewed were excluded, for reasons listed in figure 1, leaving a total of 83 studies included in this review.

We grouped the studies based on whether authors had reported efficacy or toxicity of our drugs of interest, whether the patients had HIV co-infection or not and whether patients were treated for MDR-TB or NTM infection. We further stratified studies by whether they included a control or comparison group. The numbers of studies included in each of these subgroups are summarised in figure 1 . The design and treatment characteristics of all studies included in this review are summarised in online supplementary tables S1-S7 [15-97]. Of the 25 included studies of MDR-TB, 20 were retrospective cohort studies (three of which compared one or more of our drugs of interest to a group not receiving the drug), three were prospective cohort studies (one of which compared one of our drugs of interest to a group not receiving the drug), and one was a randomised trial. Sample sizes ranged from 38 to 1027 and the average ages of the patients ranged from 28 to 45 years.

We found only one study reporting the efficacy of MDR-TB treatment with meropenem and only one study reporting toxicity of this drug used for MDR-TB. Therefore we could not perform a meta-analysis for meropenem (online supplementary material). No studies were found that reported efficacy or SAEs of imipenem-cilastatin or thioridazine. Hence, no further results are presented for meropenem, imipenemcilastatin and thioridazine.

\section{Efficacy}

We found only two controlled studies $[85,96]$ that examined the impact of AMX-CLV on treatment success in MDR-TB; as seen in table 1, the pooled RD was 0.07 (95\% CI -0.21-0.35). Results were similar in the two controlled studies $[85,96]$ that assessed the impact of clarithromycin: the pooled RD for 
treatment success was -0.13 (95\% CI -0.40-0.14). In the 36 studies of MDR-TB treatment in which authors only reported outcomes for the entire cohort, the relationship between cure rate and proportion of patients receiving macrolides (14 studies $[16,25,34,54,56,58,60,66,71,78,80,91,93]$ ), terizidone (eight studies $[34,35,40,65,76,80,83,84]$ ) or AMX-CLV (14 studies [27, 34, 54, 57, 58, 60, 66, 71, 78, $80,88,90,91,93])$ was estimated. As the proportion of patients receiving each of these drugs increased, the cure rate decreased (online supplementary fig. S1 $[16,25,27,34,35,40,54,56,58,60,65,66,71,76$, $78,80,83,84,88,90,91,93])$. In addition, the cure rate decreased as the proportion of patients with HIV infection within the study population increased (online supplementary fig. S2).

\section{Serious adverse events}

The pooled estimates for all the studies included in these meta-analyses are summarised in table 1 . There were no controlled studies of the use of azithromycin for treatment of NTM in HIV-negative patients or uncontrolled studies of azithromycin used in HIV-positive patients. As seen in figure 2a [28, 38, 39, 52, $53,64,77]$, there was significant heterogeneity between the controlled studies of toxicity of use of azithromycin for NTM in HIV-infected subjects, although only moderate heterogeneity between studies of SAEs attributed to azithromycin (fig. 2b [21, 41, 47-49]) in the uncontrolled studies of NTM treatment in HIV-uninfected patients. The estimated risk of SAEs was low in the controlled studies (pooled RD 0.04, 95\% CI -0.03-0.10) and in the uncontrolled studies of azithromycin (proportion 0.03, 95\% CI 0.00-0.08).

In HIV-infected patients treated with clarithromycin for NTM, the pooled RD for toxicity was $0.00(95 \%$ CI -0.02-0.02) in the controlled studies (fig. 3a [17, 22, 38, 42, 69, 79, 87, 95]) compared to a pooled proportion of 0.20 (95\% CI $0.12-0.27$ ) in the uncontrolled studies (fig. $3 \mathrm{~b}[18,23,26,29,31,36]$ ). In HIV-uninfected patients treated with clarithromycin for NTM (fig. 4a [44, 55, 72] and 4b [30, 32, 41, 46, $49,51,56,59,62,68,74,82,86,92,94])$, the pooled $\mathrm{RD}$ for patients experiencing a SAE in the three controlled studies (fig. 4a) was 0.01 (95\% CI -0.06-0.07), compared to a pooled SAE proportion of 0.04 (95\% CI 0.02-0.07) in the 15 uncontrolled studies (fig. 4b).

Among patients with MDR-TB, SAEs that were attributed to one of the drugs of interest were only reported for AMX-CLV in three uncontrolled studies. As seen in table 1, in these studies the pooled proportion of patients who experienced an SAE from AMX-CLV was 0.12 (95\% CI 0.00-0.28).

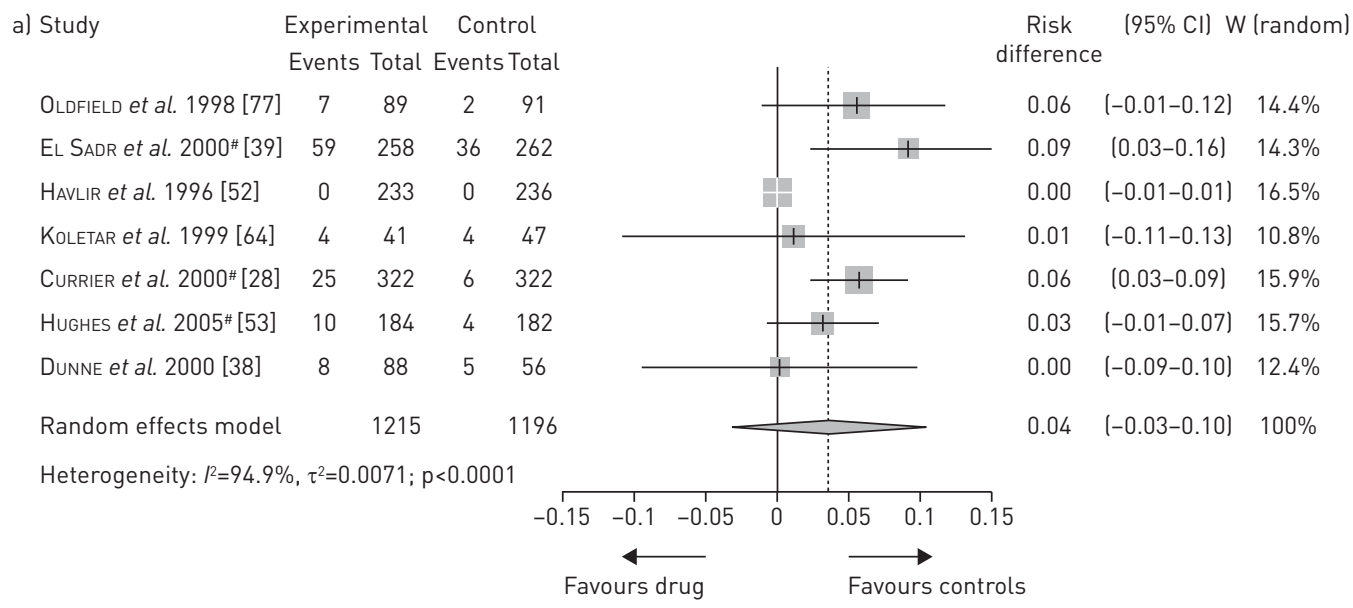

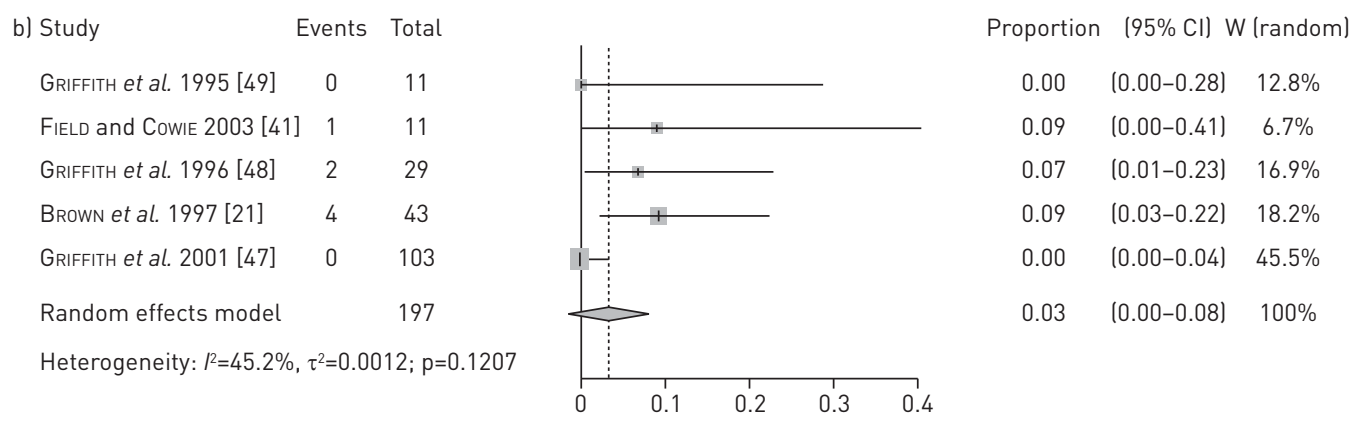

FIGURE 2 Forest plots of serious adverse events with azithromycin for treatment of non-tuberculous mycobacteria infections in al HIV-positive patients (controlled studies); b) HIV-negative patients (uncontrolled studies). \# : serious adverse events defined as grade 3-4 adverse events, or drug stopped because of adverse events. 


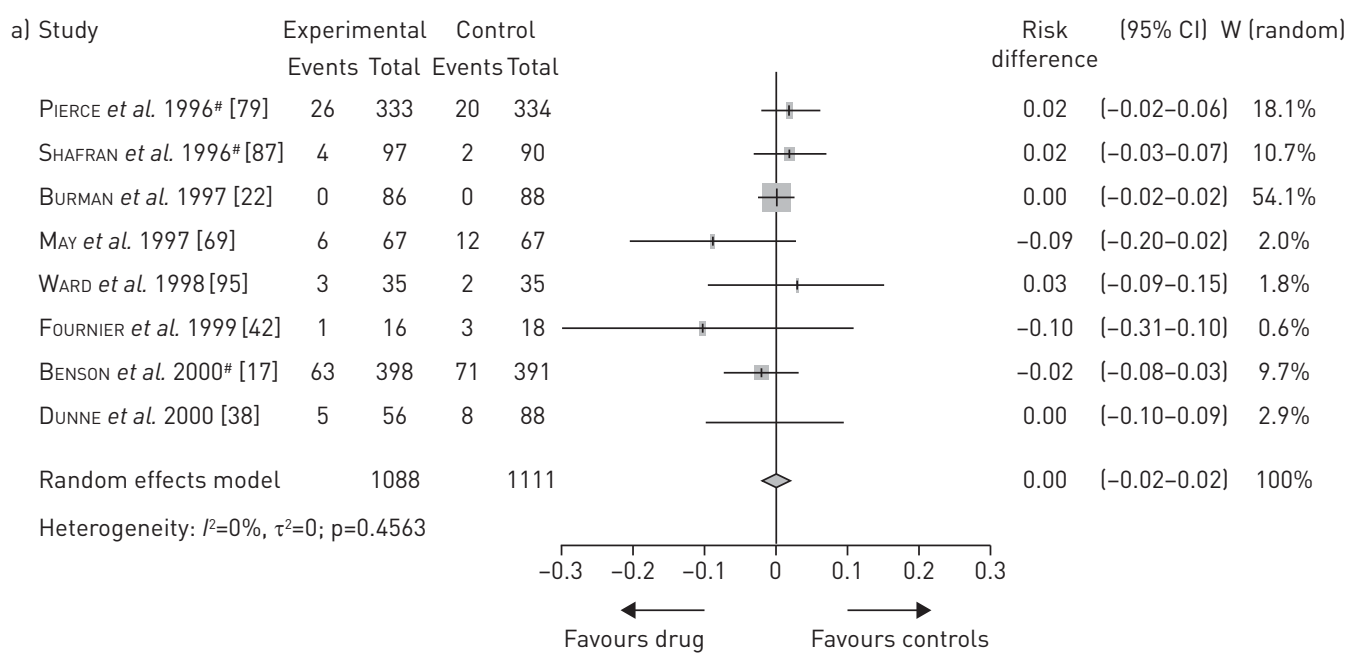

\begin{tabular}{|c|c|c|c|c|c|c|c|}
\hline Study & Events & Total & & & Proportion & $(95 \% \mathrm{CI}) \mathrm{V}$ & I Irandom \\
\hline DAUTZEnBerg et al. 1993 [29] & 5 & 77 & -1 & & 0.06 & $(0.02-0.15)$ & $18.8 \%$ \\
\hline CHaisson et al. 1994 [23] & 42 & 154 & & +1 & 0.27 & $(0.20-0.35)$ & $17.6 \%$ \\
\hline DautzenBerg et al. 1997 [31] & 15 & 55 & & +1 & 0.27 & $(0.16-0.41)$ & $13.8 \%$ \\
\hline DuBÉ et al. 1997 [36] & 8 & 53 & 1 & & 0.15 & $(0.07-0.28)$ & $15.5 \%$ \\
\hline CoHn et al. 1999\# [26] & 20 & 85 & & 1 & 0.24 & $(0.15-0.34)$ & $16.0 \%$ \\
\hline Benson et al. 2003\# [18] & 32 & 160 & & & 0.20 & $(0.14-0.27)$ & $18.3 \%$ \\
\hline Random effects model & & 584 & & & 0.20 & $(0.12-0.27)$ & $100 \%$ \\
\hline \multicolumn{8}{|c|}{ Heterogeneity: $I^{2}=82.1 \%, \tau^{2}=0.0069 ; p<0.0001$} \\
\hline & & & 0.1 & 0.3 & & & \\
\hline
\end{tabular}

FIGURE 3 Forest plots of serious adverse events attributed to clarithromycin used for treatment of non-tuberculous mycobacteria infections/disease in HIV-positive patients. al Controlled studies; b) uncontrolled studies. " : serious adverse events defined as grade 3-4 adverse events, or drug stopped because of adverse events.

\section{Discussion}

In controlled studies, HIV-infected and HIV-uninfected patients receiving macrolides for NTM infection or disease did not have a higher risk of SAEs. In uncontrolled studies the use of clarithromycin was associated with higher risk of SAE in HIV-infected patients. In three controlled studies AMX-CLV was associated with excess SAEs in MDR-TB treatment, but we found no evidence of toxicity in three studies of patients treated for NTM with AMX-CLV. Four controlled studies found no evidence of efficacy of clarithromycin or AMX-CLV for treatment of MDR-TB.

Our study had several limitations. The most important limitation was the very small number of controlled studies, and with small numbers of patients, that assessed efficacy and toxicity of only a few of the drugs of interest used for the treatment of MDR-TB. Hence we were only able to perform meta-analysis for a few of the drugs we studied. Furthermore, only one randomised trial of MDR-TB treatment was identified; the remainder were cohort studies in which patients were not allocated randomly to the regimens. Hence it is likely that the results are affected by selection bias in that more seriously ill patients, with more extensive resistance patterns may have been more likely to receive these second-line drugs.

For studies of patients treated with these drugs for NTM, the quality of studies was generally higher, particularly among HIV-infected patients as the majority of these studies were randomised trials. However, the evidence from studies of NTM treatment among HIV-uninfected patients was limited as the studies were not randomised, patients were on several drugs at once, and dosage used or length of therapy was often not reported. For the 13 RCTs included (six clarithromycin, six azithromycin and one with both drugs), the quality of the studies were moderate and most had too few patients to have adequate power to detect differences in SAEs.

Despite these limitations, our study had several strengths. We constructed a comprehensive search strategy that included RCTs and cohort studies; the latter often are conducted under conditions more 


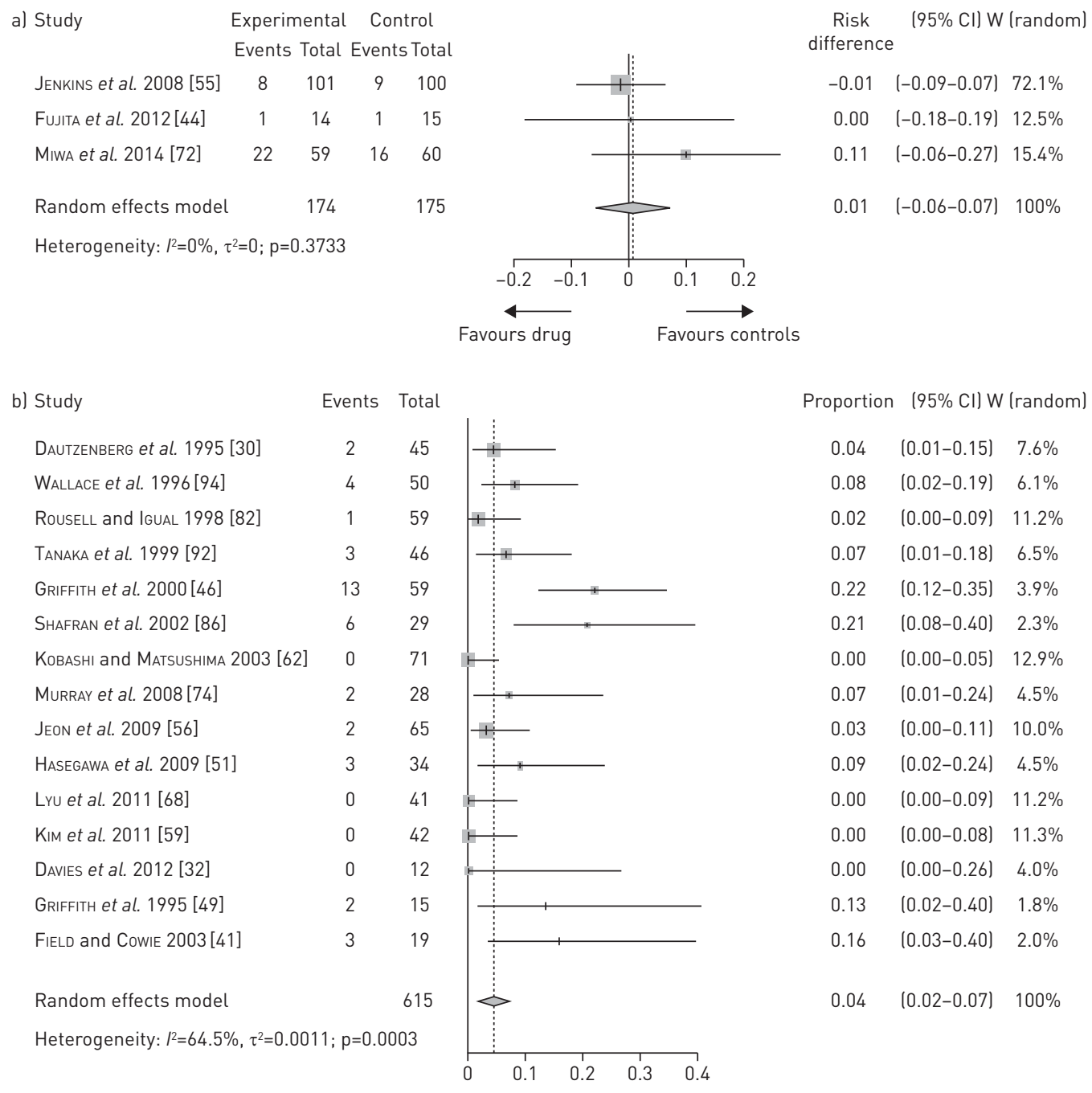

FIGURE 4 Forest plot of serious adverse events attributed to clarithromycin used for treatment of non-tuberculous mycobacteria infections/disease in HIV-negative patients. al Controlled studies; b) uncontrolled studies.

representative of clinical practice. The sensitive search strategy also resulted in a large number of articles, decreasing the likelihood that studies were missed. Additionally, we were able to stratify studies based on HIV status, allowing the interpretation of outcomes separately for each group of patients. With this stratification we were able to show that HIV-positive patients did not have any greater risk of SAEs than HIV-uninfected persons, at least in studies with stronger designs (randomised trials).

As treatment of MDR-TB is costly and requires lengthy use of many drugs, administration of safe and effective drugs is crucial. This is especially important given the potential costs of several of the drugs reviewed. We found no evidence of efficacy of any of the drugs reviewed in the few controlled studies, while in the uncontrolled cohorts, the cure rate of patients was inversely proportional to the number of patients receiving these drugs. However, this finding may be biased by the selection of sicker patients with more extensive disease and resistance to receive these second-line drugs. Although azithromycin and clarithromycin had low rates of adverse events, the lack of evidence for the efficacy of these drugs does not support their use for treatment of MDR-TB.

\section{Conclusion}

We found no evidence for efficacy of AMX-CLV, terizidone or macrolides in the treatment of MDR-TB. Although we found a low risk of toxicity with prolonged use of these drugs, the lack of evidence of their efficacy suggests a need to re-evaluate their inclusion in treatment regimens for MDR-TB. However, RCTs are required to properly assess the efficacy and safety of these drugs in the treatment of MDR-TB. 


\section{References}

World Health Organization. Global Tuberculosis Report 2014. Geneva, World Health Organization, 2014.

2 Laserson KF, Thorpe LE, Leimane V, et al. Speaking the same language: treatment outcome definitions for multidrug-resistant tuberculosis. Int J Tuberc Lung Dis 2005; 9: 640-645.

3 World Health Organization. Extensively Drug-resistant Tuberculosis (XDR-TB): Recommendations for Prevention and Control. Geneva, World Health Organization, 2006.

4 Marks SM, Flood J, Seaworth B, et al. Treatment practices, outcomes, and costs of multidrug-resistant and extensively drug-resistant tuberculosis, United States, 2005-2007. Emerg Infect Dis 2014; 20: 812-821.

5 Ahmad S, Mokaddas E. Current status and future trends in the diagnosis and treatment of drug-susceptible and multidrug-resistant tuberculosis. J Infect Public Health 2014; 7: 75-91.

6 Ahuja SD, Ashkin D, Avendano M, et al. Multidrug resistant pulmonary tuberculosis treatment regimens and patient outcomes: an individual patient data meta-analysis of 9,153 patients. PLoS Med 2012; 9: e1001300.

7 World Health Organization. Treatment of Tuberculosis Guidelines. 4th Edn. Geneva, World Health Organization, 2010.

8 Cox H, Ford N. Linezolid for the treatment of complicated drug-resistant tuberculosis: a systematic review and meta-analysis. Int J Tuberc Lung Dis 2012; 16: 447-454.

9 Sotgiu G, Centis R, D'Ambrosio L, et al. Efficacy, safety and tolerability of linezolid containing regimens in treating MDR-TB and XDR-TB: systematic review and meta-analysis. Eur Respir J 2012; 40: 1430-1442.

10 Chang KC, Yew WW, Tam CM, et al. WHO group 5 drugs and difficult multidrug-resistant tuberculosis: a systematic review with cohort analysis and meta-analysis. Antimicrob Agents Chemother 2013; 57: 4097-4104.

11 Dey $\mathrm{T}$, Brigden $\mathrm{G}$, Cox $\mathrm{H}$, et al. Outcomes of clofazimine for the treatment of drug-resistant tuberculosis: a systematic review and meta-analysis. J Antimicrob Chemother 2013; 68: 284-293.

12 Hwang TJ, Wares DF, Jafarov A, et al. Safety of cycloserine and terizidone for the treatment of drug-resistant tuberculosis: a meta-analysis. Int J Tuberc Lung Dis 2013; 17: 1257-1266.

13 Trotti A, Colevas AD, Setser A, et al. CTCAE v3.0: development of a comprehensive grading system for the adverse effects of cancer treatment. Semin Radiat Oncol 2003; 13: 176-181.

14 Higgins JP, Thompson SG. Quantifying heterogeneity in a meta-analysis. Stat Med 2002; 21: 1539-1558.

15 Hwang TJ, Wares DF, Jafarov A, et al. Safety of cycloserine and terizidone for the treatment of drug-resistant tuberculosis: a meta-analysis. Int J Tuberc Lung Dis 2013; 17: 1257-1266.

16 Anderson LF, Tamne S, Watson JP, et al. Treatment outcome of multi-drug resistant tuberculosis in the United Kingdom: retrospective-prospective cohort study from 2004 to 2007. Euro Surveill 2013; 18: 20601.

17 Benson CA, Williams PL, Cohn DL, et al. Clarithromycin or rifabutin alone or in combination for primary prophylaxis of Mycobacterium avium complex disease in patients with AIDS: a randomized, double-blind, placebo-controlled trial. J Infect Dis 2000; 181: 1289-1297.

18 Benson CA, Williams PL, Currier JS, et al. A prospective, randomized trial examining the efficacy and safety of clarithromycin in combination with ethambutol, rifabutin, or both for the treatment of disseminated Mycobacterium avium complex disease in persons with acquired immunodeficiency syndrome. Clin Infect Dis 2003; 37: 1234-1243

19 Böszörményi M. Dreifach-Blindversuch zum Vergleich der Nebenwirkungen von Terizidon (Terivalidin) und Cycloserin [Triple placebo test for comparison of side effects of terizidone (terivalidin) and cycloserine]. Int J Clin Pharmacol 1973; 8: 37-41.

20 Briones FH. Avaliacao das manifestacoes secundarias em doentes tratados com terizidona [Evaluation of secondary manifestations in patients treated with terizidone]. Rev Bras Med 1970; 27: 271-275.

21 Brown BA, Griffith DE, Girard W, et al. Relationship of adverse events to serum drug levels in patients receiving high-dose azithromycin for mycobacterial lung disease. Clin Infect Dis 1997; 24: 958-964.

22 Burman WJ, Reves RR, Rietmeijer CA, et al. A retrospective comparison of clarithromycin versus rifampin in combination treatment for disseminated Mycobacterium avium complex disease in AIDS: clarithromycin decreases transfusion requirements. Int I Tuberc Lung Dis 1997; 1: 163-169.

23 Chaisson RE, Benson CA, Dube MP, et al. Clarithromycin therapy for bacteremic Mycobacterium avium complex disease. A randomized, double-blind, dose-ranging study in patients with AIDS. Ann Intern Med 1994; 121: 905-911.

24 Chaisson RE, Keiser P, Pierce M, et al. Clarithromycin and ethambutol with or without clofazimine for the treatment of bacteremic Mycobacterium avium complex disease in patients with HIV infection. AIDS 1997; 11: 311-317.

25 Chan ED, Laurel V, Strand MJ, et al. Treatment and outcome analysis of 205 patients with multidrug-resistant tuberculosis. Am J Respir Crit Care Med 2004; 169: 1103-1109.

26 Cohn DL, Fisher EJ, Peng GT, et al. A prospective randomized trial of four three-drug regimens in the treatment of disseminated Mycobacterium avium complex disease in AIDS patients: excess mortality associated with high-dose clarithromycin. Clin Infect Dis 1999; 29: 125-133.

27 Cox HS, Kalon S, Allamuratova S, et al. Multidrug-resistant tuberculosis treatment outcomes in Karakalpakstan, Uzbekistan: treatment complexity and XDR-TB among treatment failures. PLoS One 2007; 2: e1126.

28 Currier JS, Williams PL, Koletar SL, et al. Discontinuation of Mycobacterium avium complex prophylaxis in patients with antiretroviral therapy-induced increases in $\mathrm{CD}^{+}$cell count: a randomized, double-blind, placebo-controlled trial. Ann Intern Med 2000; 133: 493-503.

29 Dautzenberg B, Saint Marc T, Meyohas MC, et al. Clarithromycin and other antimicrobial agents in the treatment of disseminated Mycobacterium avium infections in patients with acquired immunodeficiency syndrome. Arch Intern Med 1993; 153: 368-372.

30 Dautzenberg B, Piperno D, Diot P, et al. Clarithromycin in the treatment of Mycobacterium avium lung infections in patients without AIDS. Chest 1995; 107: 1035-1040.

31 Dautzenberg B, Truffot-Pernot C, Hazebroucq J, et al. A randomized comparison of two clarithromycin doses for treatment of Mycobacterium avium complex infections. Infection 1997; 25: 16-21.

32 Davies BS, Roberts CH, Kaul S, et al. Non-tuberculous slow-growing mycobacterial pulmonary infections in non-HIV-infected patients in south London. Scand J Infect Dis 2012; 44: 815-819. 
33 De Lorenzo S, Alffenaar JW, Sotgiu G, et al. Efficacy and safety of meropenem-clavulanate added to linezolid-containing regimens in the treatment of MDR-/XDR-TB. Eur Respir J 2013; 41: 1386-1392.

34 Dheda K, Shean K, Zumla A, et al. Early treatment outcomes and HIV status of patients with extensively drug-resistant tuberculosis in South Africa: a retrospective cohort study. Lancet 2010; 375: 1798-1807.

35 Diacon AH, Pym A, Grobusch MP, et al. Multidrug-resistant tuberculosis and culture conversion with bedaquiline. N Engl J Med 2014; 371: 723-732.

36 Dubé MP, Sattler FR, Torriani FJ, et al. A randomized evaluation of ethambutol for prevention of relapse and drug resistance during treatment of Mycobacterium avium complex bacteremia with clarithromycin-based combination therapy. J Infect Dis 1997; 176: 1225-1232.

37 Dumon JF, Aimone J, Dumon G. Note preliminaire sur la terizidone [Preliminary note on terizidone]. Mars Med 1972; 109: 730-732.

38 Dunne M, Fessel J, Kumar P, et al. A randomized, double-blind trial comparing azithromycin and clarithromycin in the treatment of disseminated Mycobacterium avium infection in patients with human immunodeficiency virus. Clin Infect Dis 2000; 31: 1245-1252.

39 El-Sadr WM, Burman WJ, Grant LB, et al. Discontinuation of prophylaxis against Mycobacterium avium complex disease in HIV-infected patients who have a response to antiretroviral therapy. N Engl J Med 2000; 342: $1085-1092$.

40 Ferrara G, Richeldi L, Bugiani M, et al. Management of multidrug-resistant tuberculosis in Italy. Int J Tuberc Lung Dis 2005; 9: 507-513.

41 Field SK, Cowie RL. Treatment of Mycobacterium avium-intracellulare complex lung disease with a macrolide, ethambutol, and clofazimine. Chest 2003; 124: 1482-1486.

42 Fournier S, Burguière AM, Flahault A, et al. Effect of adding clofazimine to combined clarithromycin-ethambutol therapy for Mycobacterium avium complex septicemia in AIDS patients. Eur J Clin Microbiol Infect Dis 1999; 18: 16-22.

43 Friedman ND, Athan E, Hughes AJ, et al. Mycobacterium ulcerans disease: experience with primary oral medical therapy in an Australian cohort. PLoS Negl Trop Dis 2013; 7: e2315.

44 Fujita M, Kajiki A, Tao Y, et al. The clinical efficacy and safety of a fluoroquinolone-containing regimen for pulmonary MAC disease. J Infect Chemother 2012; 18: 146-151.

45 Gordin FM, Sullam PM, Shafran SD, et al. A randomized, placebo-controlled study of rifabutin added to a regimen of clarithromycin and ethambutol for treatment of disseminated infection with Mycobacterium avium complex. Clin Infect Dis 1999; 28: 1080-1085.

46 Griffith DE, Brown BA, Cegielski P, et al. Early results (at 6 months) with intermittent clarithromycin-including regimens for lung disease due to Mycobacterium avium complex. Clin Infect Dis 2000; 30: 288-292.

47 Griffith DE, Brown BA, Girard WM, et al. Azithromycin-containing regimens for treatment of Mycobacterium avium complex lung disease. Clin Infect Dis 2001; 32: 1547-1553.

48 Griffith DE, Brown BA, Girard WM, et al. Azithromycin activity against Mycobacterium avium complex lung disease in patients who were not infected with human immunodeficiency virus. Clin Infect Dis 1996; 23: 983-989.

49 Griffith DE, Brown BA, Girard WM, et al. Adverse events associated with high-dose rifabutin in macrolide-containing regimens for the treatment of Mycobacterium avium complex lung disease. Clin Infect Dis 1995; 21: 594-598.

50 Hammerli A, Gallindo IS. Tratamento da tuberculose cronica resistente. Um novo tuberculostatico: terizidona. [Treatment of resistant chronic tuberculosis. A new antitubercular agent: terizidone.] Rev Bras Med 1969; 26: 684-688.

51 Hasegawa N, Nishimura T, Ohtani S, et al. Therapeutic effects of various initial combinations of chemotherapy including clarithromycin against Mycobacterium avium complex pulmonary disease. Chest 2009; 136: 1569-1575.

52 Havlir DV, Dubé MP, Sattler FR, et al. Prophylaxis against disseminated Mycobacterium avium complex with weekly azithromycin, daily rifabutin, or both. N Engl J Med 1996; 335: 392-398.

53 Hughes WT, Dankner WM, Yogev R, et al. Comparison of atovaquone and azithromycin with trimethoprim-sulfamethoxazole for the prevention of serious bacterial infections in children with HIV infection. Clin Infect Dis 2005; 40: 136-145.

54 Isaakidis $\mathrm{P}$, Varghese B, Mansoor H, et al. Adverse events among HIV/MDR-TB co-infected patients receiving antiretroviral and second line anti-TB treatment in Mumbai, India. PLoS One 2012; 7: e40781.

55 Jenkins PA, Campbell IA, Banks J, et al. Clarithromycin vs ciprofloxacin as adjuncts to rifampicin and ethambutol in treating opportunist mycobacterial lung diseases and an assessment of Mycobacterium vaccae immunotherapy. Thorax 2008; 63: 627-634.

56 Jeon DS, Kim DH, Kang HS, et al. Survival and predictors of outcomes in non-HIV-infected patients with extensively drug-resistant tuberculosis. Int J Tuberc Lung Dis 2009; 13: 594-600.

57 Jeon K, Kwon OJ, Lee NY, et al. Antibiotic treatment of Mycobacterium abscessus lung disease: a retrospective analysis of 65 patients. Am J Respir Crit Care Med 2009; 180: 896-902.

58 Jiang $\mathrm{RH}, \mathrm{Xu} \mathrm{HB}, \mathrm{Li}$ L. Comparative roles of moxifloxacin and levofloxacin in the treatment of pulmonary multidrug-resistant tuberculosis: a retrospective study. Int J Antimicrob Agents 2013; 42: 36-41.

59 Kim EY, Chi SY, Oh IJ, et al. Treatment outcome of combination therapy including clarithromycin for Mycobacterium avium complex pulmonary disease. Korean J Intern Med 2011; 26: 54-59.

60 Kim HR, Hwang SS, Kim HJ, et al. Impact of extensive drug resistance on treatment outcomes in non-HIV-infected patients with multidrug-resistant tuberculosis. Clin Infect Dis 2007; 45: 1290-1295.

61 Kobashi Y, Abe M, Mouri K, et al. Relationship between clinical efficacy for pulmonary MAC and drug-sensitivity test for isolated MAC in a recent 6-year period. J Infect Chemother 2012; 18: 436-443.

62 Kobashi Y, Matsushima T. The effect of combined therapy according to the guidelines for the treatment of Mycobacterium avium complex pulmonary disease. Intern Med 2003; 42: 670-675.

63 Kobashi Y, Matsushima T, Oka M. A double-blind randomized study of aminoglycoside infusion with combined therapy for pulmonary Mycobacterium avium complex disease. Respir Med 2007; 101: 130-138.

64 Koletar SL, Berry AJ, Cynamon MH, et al. Azithromycin as treatment for disseminated Mycobacterium avium complex in AIDS patients. Antimicrob Agents Chemother 1999; 43: 2869-2872.

65 Kvasnovsky CL, Cegielski JP, Erasmus R, et al. Extensively drug-resistant TB in Eastern Cape, South Africa: high mortality in HIV-negative and HIV-positive patients. J Acquir Immune Defic Syndr 2011; 57: 146-152. 
Leimane V, Dravniece G, Riekstina V, et al. Treatment outcome of multidrug/extensively drug-resistant tuberculosis in Latvia, 2000-2004. Eur Respir J 2010; 36: 584-593.

67 Lindeboom JA, Kuijper EJ, Bruijnesteijn van Coppenraet ES, et al. Surgical excision versus antibiotic treatment for nontuberculous mycobacterial cervicofacial lymphadenitis in children: a multicenter, randomized, controlled trial. Clin Infect Dis 2007; 44: 1057-1064.

68 Lyu J, Jang HJ, Song JW, et al. Outcomes in patients with Mycobacterium abscessus pulmonary disease treated with long-term injectable drugs. Respir Med 2011; 105: 781-787.

69 May T, Brel F, Beuscart C, et al. Comparison of combination therapy regimens for treatment of human immunodeficiency virus-infected patients with disseminated bacteremia due to Mycobacterium avium. Clin Infect Dis 1997; 25: 621-629.

70 Micheletti V, Teti V, Fenzi P, et al. [The therapy of pulmonary tuberculosis in psychopathic patients, using terizidone]. Minerva Med 1968; 59: 2459-2465.

71 Mitnick CD, Shin SS, Seung KJ, et al. Comprehensive treatment of extensively drug-resistant tuberculosis. $N$ Engl J Med 2008; 359: 563-574.

72 Miwa S, Shirai M, Toyoshima M, et al. Efficacy of clarithromycin and ethambutol for Mycobacterium avium complex pulmonary disease: a preliminary study. Ann Am Thorac Soc 2014; 11: 23-29.

73 Gonzalez Montaner LJ, Dambrosi A, Manassero M, et al. Adverse effects of antituberculosis drugs causing changes in treatment. Tubercle 1982; 63: 291-294.

74 Murray MP, Laurenson IF, Hill AT. Outcomes of a standardized triple-drug regimen for the treatment of nontuberculous mycobacterial pulmonary infection. Clin Infect Dis 2008; 47: 222-224.

75 O'Brien DP, McDonald A, Callan P, et al. Successful outcomes with oral fluoroquinolones combined with rifampicin in the treatment of Mycobacterium ulcerans: an observational cohort study. PLoS Negl Trop Dis 2012; 6: e1473.

76 O'Donnell MR, Padayatchi N, Kvasnovsky C, et al. Treatment outcomes for extensively drug-resistant tuberculosis and HIV co-infection. Emerg Infect Dis 2013; 19: 416-424.

77 Oldfield EC III, Fessel WJ, Dunne MW, et al. Once weekly azithromycin therapy for prevention of Mycobacterium avium complex infection in patients with AIDS: a randomized, double-blind, placebo-controlled multicenter trial. Clin Infect Dis 1998; 26: 611-619.

78 Palacios E, Dallman R, Muñoz M, et al. Drug-resistant tuberculosis and pregnancy: treatment outcomes of 38 cases in Lima, Peru. Clin Infect Dis 2009; 48: 1413-1419.

79 Pierce M, Crampton S, Henry D, et al. A randomized trial of clarithromycin as prophylaxis against disseminated Mycobacterium avium complex infection in patients with advanced acquired immunodeficiency syndrome. $N$ Engl J Med 1996; 335: 384-391.

80 Pietersen E, Ignatius E, Streicher EM, et al. Long-term outcomes of patients with extensively drug-resistant tuberculosis in South Africa: a cohort study. Lancet 2014; 383: 1230-1239.

81 Rizzo A, Gomes A, Antunes G, et al. Cicloserina e terizidona no tratamento da tuberculose pulmonar: estudo clinico da tolerancia [Cycloserine and terizidone in the treatment of pulmonary tuberculosis: clinical study of tolerance]. Rev Bras Med 1969; 26: 119-122.

82 Roussel G, Igual J. Clarithromycin with minocycline and clofazimine for Mycobacterium avium intracellulare complex lung disease in patients without the acquired immune deficiency syndrome. Int J Tuberc Lung Dis 1998; 2: $462-470$

83 Seddon JA, Hesseling AC, Godfrey-Faussett $\mathrm{P}$, et al. High treatment success in children treated for multidrug-resistant tuberculosis: an observational cohort study. Thorax 2014; 69: 458-464.

84 Seddon JA, Hesseling AC, Willemse M, et al. Culture-confirmed multidrug-resistant tuberculosis in children: clinical features, treatment, and outcome. Clin Infect Dis 2012; 54: 157-166.

85 Seung KJ, Becerra MC, Atwood SS, et al. Salvage therapy for multidrug-resistant tuberculosis. Clin Microbiol Infect 2014; 20: 441-446.

86 Shafran I, Kugler L, El-Zaatari FA, et al. Open clinical trial of rifabutin and clarithromycin therapy in Crohn's disease. Dig Liver Dis 2002; 34: 22-28.

87 Shafran SD, Singer J, Zarowny DP, et al. A comparison of two regimens for the treatment of Mycobacterium avium complex bacteremia in AIDS: rifabutin, ethambutol, and clarithromycin versus rifampin, ethambutol, clofazimine, and ciprofloxacin. N Engl J Med 1996; 335: 377-383.

88 Shin SS, Pasechnikov AD, Gelmanova IY, et al. Adverse reactions among patients being treated for MDR-TB in Tomsk, Russia. Int J Tuberc Lung Dis 2007; 11: 1314-1320.

89 Shmelev NA, Shabalova LN, Kolosovskaia VP. Sravnitel'noe kliniko-elektorentsefalograficheskoe izuchenie perenosimosti tsikloserina i terizidona [Comparative clinical electroencephalographic study of cycloserine and terizidone tolerance]. Antibiotiki 1975; 20: 174-180.

90 Singla R, Caminero JA, Jaiswal A, et al. Linezolid: an effective, safe and cheap drug for patients failing multidrug-resistant tuberculosis treatment in India. Eur Respir J 2012; 39: 956-962.

91 Tahaoğlu K, Törün T, Sevim T, et al. The treatment of multidrug-resistant tuberculosis in Turkey. N Engl J Med 2001; 345: 170-174.

92 Tanaka E, Kimoto T, Tsuyuguchi K, et al. Effect of clarithromycin regimen for Mycobacterium avium complex pulmonary disease. Am J Respir Crit Care Med 1999; 160: 866-872.

93 Törün T, Güngör G, Ozmen J, et al. Side effects associated with the treatment of multidrug-resistant tuberculosis. Int J Tuberc Lung Dis 2005; 9: 1373-1377.

94 Wallace RJ Jr, Brown BA, Griffith DE, et al. Clarithromycin regimens for pulmonary Mycobacterium avium complex. The first 50 patients. Am J Respir Crit Care Med 1996; 153: 1766-1772.

95 Ward TT, Rimland D, Kauffman C, et al. Randomized, open-label trial of azithromycin plus ethambutol vs. clarithromycin plus ethambutol as therapy for Mycobacterium avium complex bacteremia in patients with human immunodeficiency virus infection. Clin Infect Dis 1998; 27: 1278-1285.

$96 \mathrm{Xu} \mathrm{HB}$, Jiang RH, Xiao HP. Clofazimine in the treatment of multidrug-resistant tuberculosis. Clin Microbiol Infect 2012; 18: 1104-1110

97 Zrilić V, Mijatović M, Mirković S, et al. Rezultati klinickog ispitivanja novog antituberkulotika terizidona (Terivalidina) [Results of clinical trials of a new antitubercular agent, terizidone (Terivalidine)]. Plucne Bolest $i$ Tuberk 1972; 24: 89-98. 